\title{
Panel on Some Issues on Testing Theory and its Applications
}

\author{
Ana Cavalli \\ Institut National des Telecommunications \\ 9, rue Charles Fourier, 91011 Evry Cedex, France
}

The participants to the panel were Gregor v. Bochmann (University of Montreal, CA), Claude Jard (Irisa, FR), Jan de Meer (GMD, G), Kazuhito Ohmaki (ETL, JP), Alexandre Petrenko (Universite de Montreal, CA) and myself.

The discussion was focused on the following aspects of testing theory and its applications :

- how to obtain testable specifications and to insure full coverage of faults given sound assumptions on implementations,

- how to take into account the change in the profile of applications,

- how to apply the techniques developed for protocol testing to software testing,

- how to integrate verification techniques to testing, and

- to evaluate the experience on the application of formal methods to real protocols.

The discussion was aimed at addressing the problem of how to obtain testable specifications from untestable ones. And to make them tractable by formal methods and to guarantee a full coverage of faults. It was mentioned that the transformation of specifications in both formal models, Labelled Transition Systems (LTSs) and Finite State Machines (FSMs) is based on the assumptions that these models be deterministic, completely specified, strongly connected and minimal. Even with these assumptions, two problems remain to be solved: the upper bound on the number of states of the implementation and the length of test sequences. Moreover, in the case of a nondeterministic global FSM, no answer is given to the kind of faults detected.

It was mentioned also that the profiles of applications have changed in recent years, introducing new issues such as service validation, ODP testing, ATM networks. This fact indicated the need to extend the existing testing techniques to cope with these new applications.

The main focus of interest for some panelists was the application of techniques of protocol testing theory to the area of software testing, as for example, to the test of reactive systems, real time systems and distributed system management. As an example, it was mentioned the application of methods based on Extended Finite State Machines (state identification techniques including values of local variables) to the test of reactive systems.

Suggestions were made to combine the application of verification techniques with testing techniques. It could be useful to use some specifications languages as, for example, temporal logic, to describe test purposes. It would be interesting also to use some results from the area of distributed debugging.

As an experience on application of formal methods to real protocols, it was mentioned the work performed by INTAP,JP. INTAP coordinates a Formal Description Techniques group, that includes industrial enterprises as Mitsubishi, Toshiba, NEC, Hitachi, Fujitsu, Sharp, Matsushita, etc. This group intends to apply FDTs to real protocols. It was raised the difficulty to formalize test purposes, and particularly, intents of users.

Finally the discussion outlined the following orientations for future work on testing: to extend the application of protocol testing to other applications and to the area of software testing and to combine application of verification techniques with testing techniques. 